\title{
Load Balancing Applying Resources Based on Runtime Conditions in Cloud Computing
}

\author{
Bhisham Bhanot, Amit Jain
}

\begin{abstract}
With the fast advancement of the Internet, numerous sellers have begun to give cloud administrations. An everincreasing number of administrations can be gotten in the cloud, and clients don't need to do activities on a nearby PC. All activities are figured on the cloud. At the point when an enormous number of clients endeavor to access cloud benefits, this frequently makes the server neglect to react. Deciding a technique by which to give clients auspicious and exact reactions is a subject deserving of cutting edge examine. A few examinations have been proposed to assess and to create calculations and load adjusting approaches for cloud-based applications. It is hard for a server to manage the progression of data created by the majority of the different endeavors endeavoring to get to it. Unreasonable stream causes server over-load with a resulting loss of data. So the primary target of this examination is to investigate the outstanding task at hand among every single virtual machine and execute upgraded load adjusting the calculation to increase the allotment of VM to cloudlet and limit the make span time.
\end{abstract} time.

Index Terms:. cloud infrastructure, load balancing, makespan

\section{INTRODUCTION}

Highlight a section that you want to designate with a certain style, and then select the appropriate name on the style menu. The style will adjust your fonts and line spacing. Do not change the font sizes or line spacing to squeeze more text into a limited number of pages. Use italics for emphasis; do not underline.

The idea of distributed computing is utilized on-request access to common and configurable handling resources, for instance, applications and administrations (PaaS), working frameworks (PaaS), servers (IaaS), that can be made open and settled with a decreased measure of affiliation exercises or individual agreeable fenced in areas. In a brief time span, circulated processing has been associated extensively in various applications, it transformed into an essential bit of the cutting-edge period of enlisting system expecting next to zero exertion, that engages customers to utilize their advantages as a pay investigate as portrayed in Figure 1.

\section{A. The demand for Load Balancing in the Cloud Computing}

It [1] is a strategy with the true objective that it doles out the remaining task at hand likewise among all the open center points which are accessible in the system. Higher customer

Revised Manuscript Received on June 15, 2019.

Bhisham Bhanot, Comupter Science \& Engineering, L.C.E.T,

Ludhiana, India.

Amit Jain, Comupter Science \& Engineering, L.C.E.T,

Ludhiana, India. satisfaction is the saying behind load altering. As the amount of customer's and moreover their solicitations are extending well ordered, the mists should outfit the organizations to the customers with their for the most part satisfaction. An appropriate or an ideal load adjusting figuring help in making use of the available resources most decidedly, along these lines ensuring no center is under-stacked or over-stacked. Load adjusting approves versatility, ambiguities robberies and furthermore diminishes time. A few load adjusting count [2] have been arranged remembering the true objective to design the stack among various machines. In any case, so far there is no such immaculate load changing count has been created which will convey the pile consistently over the system. It has been exhibited that conveying the errands consistently over the system is believed to be an NP-complete issue [3].

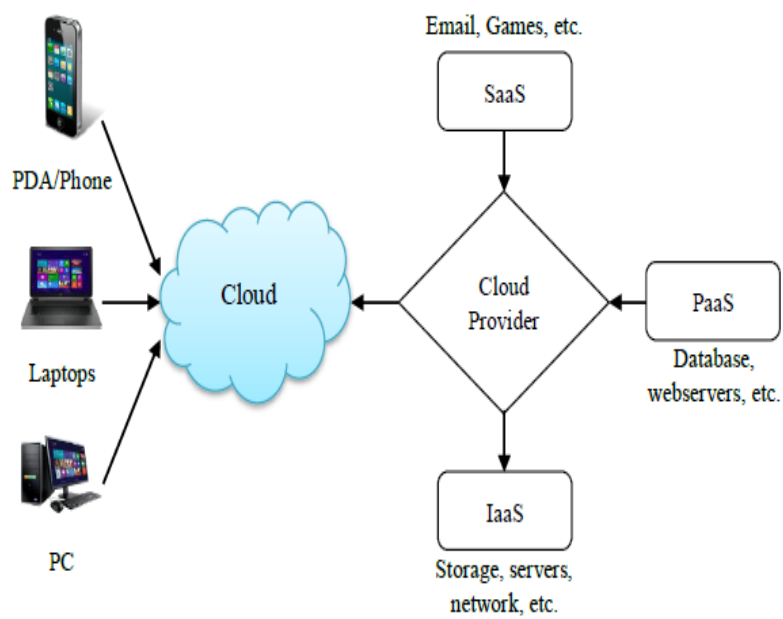

Figure 1: Cloud Computing Overview

\section{LITERATURE SURVEY}

T. Kumar et al. (2015) [4] surveyed throttled stack altering figuring. They exhibit that the throttled stack altering methodology gives the best all things considered response time layout and server ranch planning time with small taking care of incurred significant damage by using the ORT advantage delegate approach and some change parameters when appeared differently in relation to other load adjusting figuring.

Gopinath et al. (2015) [5] presented an expansive examination through the execution of the two load altering computations explicitly MaxMin and Min-Min in light of their picked cloud condition. They exhibit that

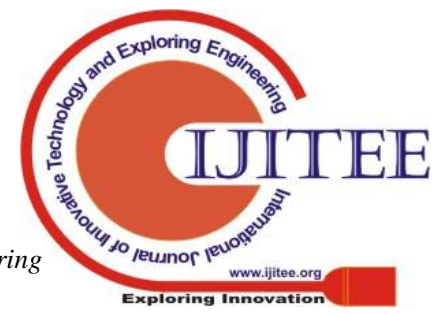


the Max-Min performs better than anything Min-Min to the extent make-length.

S. Jain et al. (2016) [6] examined different sorts of LB calculations which help to adjust the heap. In their paper, they talk a few calculations like Ant Colony Optimization, Max-Min, Round-Robin, Biased Random Sampling and Min-Min calculation with the goal that they can discover which one is best for adjusting the heap.

O. Kaneria et al. (2016) [7] proposes a proficient calculation for dispersion of assignment on the particular server farms. It checks for the accessibility of hosts on the server farms just as the preparing components and assigns the errand in like manner. Their point is to recognize the accessible server farms and recursively scan for the accessibility of preparing components.

R. K. Jena et al. (2017) [8] showed multi-objective CSA based progression estimation which can deal with the task booking issue under the enrolling condition, whereof the amount of server ranch and customer work changes intensely. Nevertheless, in advancing condition, circulated figuring resources ought to be worked in a perfect world manner. Thusly, multi-objective CSA based count is suitable for conveyed processing condition in light of the fact that the figuring can effectively utilize the structure advantages for decrease imperativeness and make-range.

S. Subha et al. (2017) [9] present a multi-target cross breed Fruitfly streamlining strategy dependent on SA for load adjusting in distributed computing situations. They have done two examinations. The main examination contrasts the first FOA and the Hybrid FOA approach and the second part reproduces the FOA-SA-LB and contrasts the outcome and the current metaheuristic methodologies, for example, PSO, HBB-LB, and EFOA-LB. The proposed FOA-SA-LB beats the first FOA downsides and achieves ideal answer for adjusting the remaining loads among virtual machines viable.

T. Smadi et al. (2017) [10] exhibited a variable administration merchant steering strategy for the preparing and reaction time of the client's solicitations to limit inside an adequate scope of expense. The proposed approach

changes the arranging and determination conditions of the old arrangement.

S. Başaran et al. (2017) [11] exhibited the examination that demonstrates a developing increment in light of a legitimate concern for delicate figuring procedures to cloud. The scholarly network ought to be urged to lead increasingly pragmatic investigations and the business to work to see that these proposed systems are actualized to build up a strong establishment for improving delicate figuring methods in the cloud conditio

A. Gherbiet al. (2018) [12] present a methodology utilized in distributed computing to dispense errands to VMs and accomplish an abnormal state of QoS by augmenting the used assets and limiting the number of utilized assets of the DC will spare the expense and vitality utilization.

Additionally, utilizing characterization for VMs as rates is certifiably not an intricate technique and will decrease the expense and time, and there is no loss of procedure time for looking to locate an accessible VM. At long last, utilizing supporting hub will diminish the outstanding load on LoadBalancer and sharing a few assignments in the equivalent virtual machine will lessen the rejected errands.

B. Kaur et al. (2018) [13] talks about the multifaceted nature of asset the executives in the cloud in contrast with different situations. The significance of planning and load adjusting in cloud condition alongside the essentialness of heuristics and metaheuristics to enhance the usage of assets and improve the exhibition of VMs has additionally talked about.

\section{ALGORITHM USED FOR THE LOAD BALANCING}

Load Balancing is accomplished in cloud condition in two stages: the initial one is to circulate the undertaking among the hub, the second one is to screen the virtual machine and play out the heap adjusting activity utilizing task movement or virtual machine relocation approach. The point of errand booking is to make a timetable and doled out each assignment to the hub (virtual machine) for explicit timespan with the goal that all undertaking are executed in the least time range. Undertaking planning is NP finished issue in the field of software engineering since a number of errand and length of assignment change in all respects quickly in cloud condition. It is hard to figure all conceivable errand asset mapping in cloud condition and locate an ideal mapping isn't a simple assignment. In this way, we need a proficient assignment planning calculation that can disseminate the errand in a successful manner so less number of virtual machine ought to be in overload or under stacked condition. In the wake of designating the assignment to a virtual machine, cloud task scheduler begin to perform load adjusting activity with the goal that undertaking can be move from the over-load virtual machine to under stacked virtual machine and all virtual machines ought to stay in equalization condition.

Following are the means for load adjusting:

Stage 1: Create a cloud condition, and produce both cloudlets and virtual machines.

Stage 2: Assign the virtual machines to the cloudlets for (int $\mathrm{I}=0$; $\mathrm{I}<$ measure; $\mathrm{i}++$ )

Stage 3: After allotting, register the heap and limit of every virtual machine

\section{Stage 4: Then check If( Load_VM>capacity_VM)}

i. Load Balancing activity is absurd

ii. Use Elasticity

iii. Go to stage 8

Stage 5: Find the quantity of under stacked, adjusted and over-load Virtual Machine. 
i. $\quad$ Then locate the under stacked, over-load and adjust machines.

ii. //UM, OM are variable $\mathrm{UM}=0.25 *, \mathrm{OM}=0.8^{*}$

Stage 6: Sort the Over stacked machines in sliding request and Under stacked machines in the climbing request.

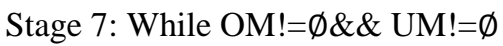

For circle for OM, In the event that OM and UM is existed, at that point move the assignment from OM to UM

\section{$T \rightarrow V \mid$ Until load at $V \leq \mathrm{OM} \| V \geq \mathrm{UM}$}

Additionally figure move time of assignment (T/data transfer capacity).

Stage 8: After the status of each VM is checked by the errand move, in the event that there is any virtual machine still in an overload condition, at that point recreate load adjusting activity some other time [14].

\section{RESULTS}

Following are the diagrams that demonstrate the normal usage of a number of the overstacked machine in essential load offsetting calculation concerning improved load adjusting calculation.. Likewise, move time has additionally been improved in proposed load adjusting calculation

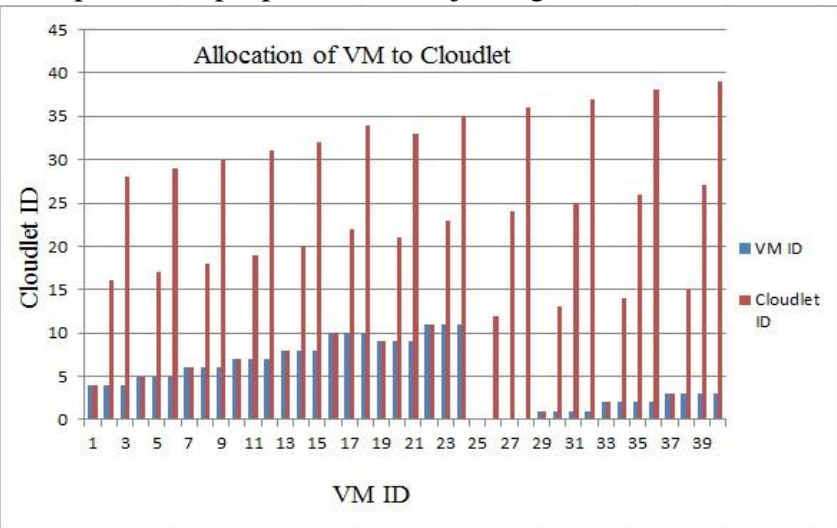

Figure 3: Allocation of VM to Cloudlet

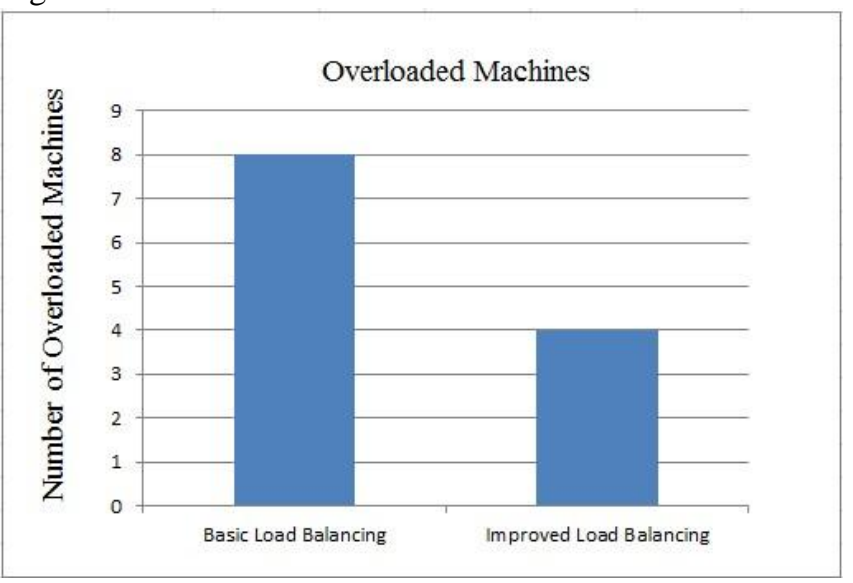

Figure 4: Number of overloaded machines

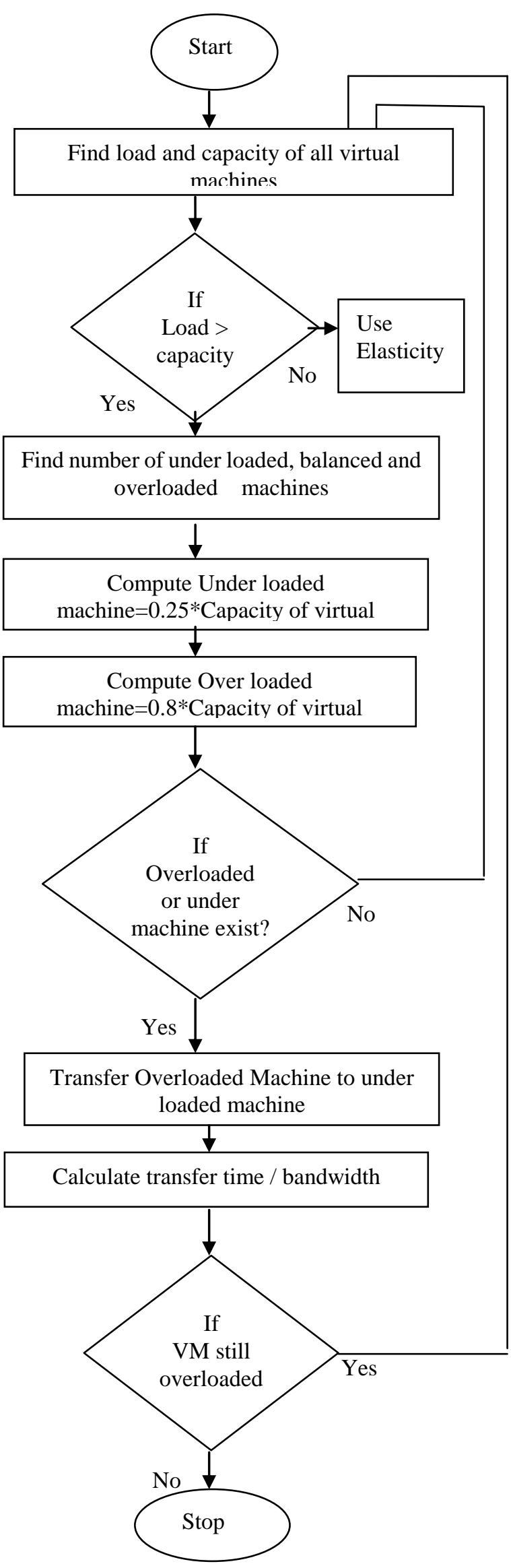

Figure 2: Flow chart of load balancing algorithm 


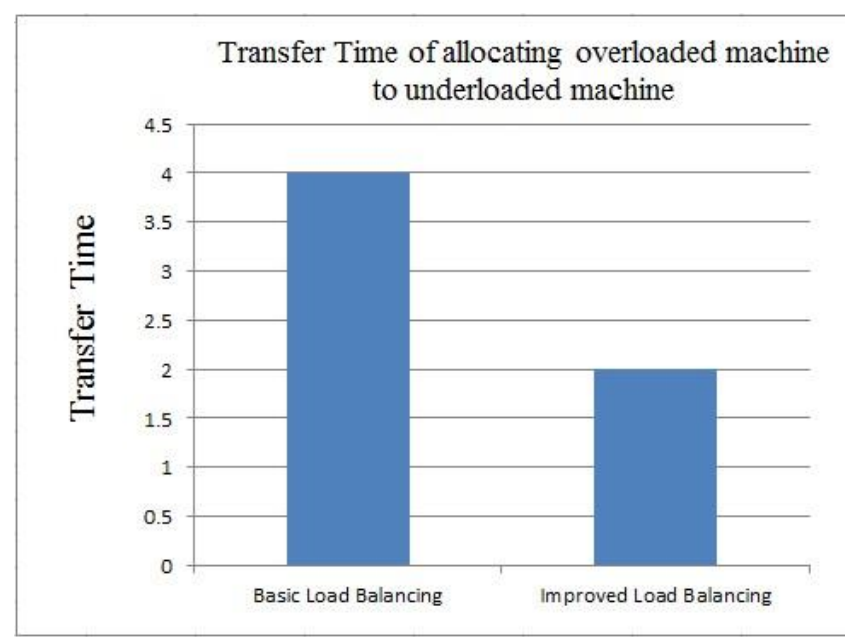

Figure 5: Transfer time of allocating overloaded machine to underloaded machine

Figure 6: Response Time of Basic and improved Algorithm

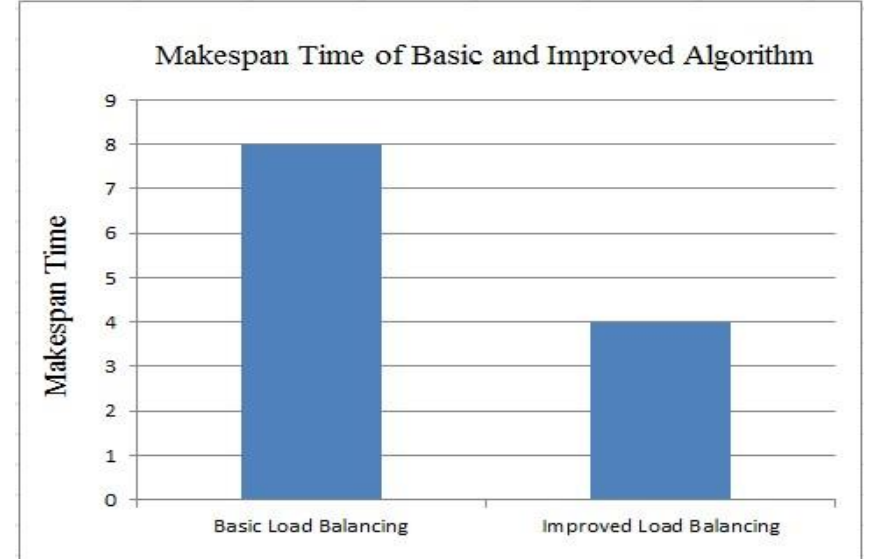

Figure 7: Makespan Time of basic and improved Algorithm.

\section{CONCLUSION}

In this examination, another cloud load adjusting components is proposed by looking at past investigations. The proposed new worldview for load adjusting engineering and a calculation can be connected to both virtual web servers and physical servers. The calculation is created so as to lessen the holding up time and make-length time; just as devalued the quantity of over-load and under stacked machines. This is finished by relegating under stacked machines to over-load machines after the process the quantity of the two machines. We have built up another and proficient Improved Load Balancing technique to decrease the reaction time, move

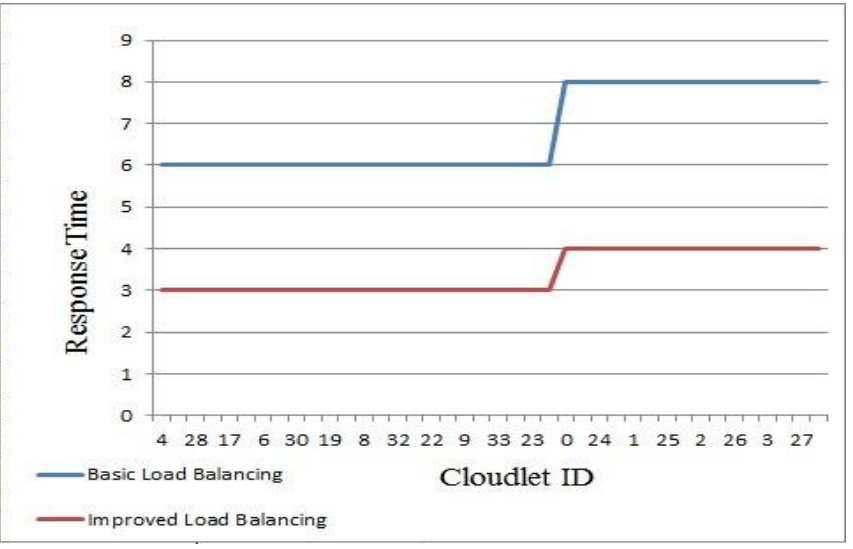

time, and a number of over-load machines. This is finished by allotting free handling components. Test results demonstrate that the improved load adjusting calculation expands the general normal asset usage proportion as a contrast with the fundamental load adjusting calculation

\section{References}

1. N. Ajith Singh, M. Hemalatha, "An approach on semi distributed load balancing algorithm for cloud computing systems" International Journal of Computer Applications Vol-56 No.12 2012

2. Shanti Swaroop moharana, Rajadeepan d. Ramesh \&DigamberPowar, "Analysis of load balancers in cloudcomputing" International Journal of Computer Science and Engineering (IJCSE) ISSN 2278-9960 Vol 2, Issue2, May 2013, 101-108.

3. D. Fernández-Baca: Allocating modules to processors in a distributed system, IEEE Transactions onSoftware Engineering, Vol. 15, No. 11, pp. 1427-1436 (1989

4. Vibhore Tyagi, Tarun Kumar, "ORT Broker Policy: Reduce Cost and Response Time Using Throttled LoadBalancing Algorithm" in Procedia Computer Science Volume 48, 2015, Pages 217-221

5. P.P. Geethu Gopinath, Shriram K. Vasudevan, "An In-depth Analysis and Study of Load BalancingTechniques in the Cloud Computing Environment" in Procedia Computer Science Volume 50, 2015, Pages 427-432

6. S. Jain and A. K. Saxena, "A survey of load balancing challenges in a cloud environment," 2016International Conference System Modeling \& Advancement in Research Trends (SMART), Moradabad, 2016, pp. 291-293

7. O. Kaneria and R. K. Banyal, "Analysis and improvement of load balancing in Cloud Computing," 2016International Conference on ICT in Business Industry \& Government (ICTBIG), Indore, 2016, pp $1-5$

8. R. K. Jena, "Energy Efficient Task Scheduling in Cloud Environment" in Energy Procedia, Volume 141,December 2017, Pages 222-227

9. M. Lawanyashri, Dr. Balamurugan Balusamy, Dr. S. Subha, "Energy-aware Hybrid Fruitfly Optimizationfor load balancing in cloud environments for EHR applications" in Informatics in Medicine Unlocked Volume8, 2017, Pages 42-50

10. Ahmed M. Manasrah, Tariq Smadi, Ammar ALmomani, "A Variable Service Broker Routing Policy for data center selection in cloud analyst" in Journal of King Saud University - Computer and Information Sciences Volume 29, Issue 3, July 2017, Pages 365-37

11. Obinna H. Ejimogu, SerenBaşaran, "A systematic mapping study on soft computing techniques to cloud environment" in Procedia Computer Science Volume 120, 2017, Pages 31-38

12. Mousa Elrotub, Abdelouahed Gherbi, "Virtual Machine Classification-based Approach to Enhanced Workload Balancing for Cloud Computing Applications" in Procedia Computer Science Volume 130, 2018, Pages 683-688

13. Amanpreet Kaur, Bikrampal Kaur, Dheerendra Singh, " Meta-heuristic based framework for workflow load balancing in cloud environment " in International Journal of Information Technology, 2018, pp 1-7

14. Mohit Kumar, S. C. Sharma, "Dynamic load balancing algorithm for balancing the workload among virtualmachine in cloud computing" in Procedia Computer Science Volume 115, 2017, Pages 322-329

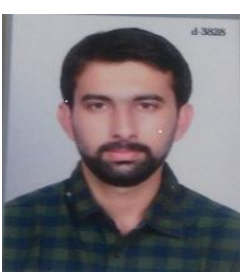

\section{Author Profile}

Bhisham Bhanot research scholar M.tech final year, Computer Science \& Engineering, L.C.E.T Ludhiana, India

Published By: 


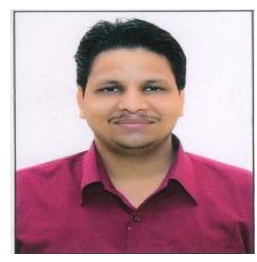

Er. Amit Jain, Associate Professor, Department of Computer Science \& Enginreering, L.C.E.T Ludhiana, India 